\title{
Tumour Suppressors and Cellular Senescence
}

Adelyne S. L. Chan, Sophia N. Mowla, Parineeta Arora and Parmjit S. Jat

Department of Neurodegenerative Disease and MRC Prion Unit

UCL Institute of Neurology

Queen Square

London WC1N 3BG

UK

Corresponding author: Parmjit S. Jat

Telephone: 442078373973

FAX: 442076762180

E-mail: p.jat@prion.ucl.ac.uk

Key words: Cellular senescence, tumour suppression, stable growth arrest, telomere shortening, aging 


\section{Abstract}

Cellular senescence is a stable cell cycle arrest that normal cells undergo in response to a variety of intrinsic and extrinsic stimuli including: progressive telomere shortening, changes in telomeric structure, or other forms of genotoxic as well non-genotoxic stress. Senescence is thought to have originated as a remodelling program that is active in embryonic development, and acts as a key tumour suppressor mechanism during the reproductive stage in early adult life, by leading to the removal of potentially cancerous cells. However, in later adult life, it promotes organismal aging by compromising tissue repair and regeneration due to the accumulation of senescent cells, depletion of stem/ progenitor cells and secretion of an array of inflammatory cytokines, chemokines and matrix metalloproteases. Whilst suppressing tumour formation in the senescent cells, these inflammatory cytokines, chemokines and metalloproteases can promote tumour progression and metastasis in the neighbouring cells. Herein, we review the molecular pathways that underlie cellular senescence and how it contributes towards tumour suppression. 


\section{Introduction}

Cellular senescence was discovered by Leonard Hayflick in 1960's when he observed that normal human cells cultured in vitro have a limited replicative capacity, referred to as the "Hayflick limit" \{HAYFLICK, 1961198 /id\}. When he exposed normal human embryonic cells to cancer cell extracts in the hope of eliciting cancer-like changes required for tumorigenesis, he observed that normal cells no longer grew after being passaged extensively in vitro. Further experiments demonstrated that cells in culture underwent a relatively consistent number of cell doublings before undergoing a cell cycle arrest. This non-dividing state is known as senescence and is induced in human fibroblasts after $50-80$ population doublings depending on the culture conditions and exposure to stress. At the time, the concept that cells would only undergo a limited number of cell doublings in vitro was highly controversial, as cells were thought to be capable of indefinite growth \{Shay, 2000199 /id\}.

Cellular senescence limits replicative capacity in vitro and in vivo. This phenomenon was initially associated with the shortening of telomeres at each division, as somatic cells are unable to replicate the very ends of linear DNA molecules due to the "end replication problem" \{Harley, 1990219 /id\}. As a result, the ends of chromosomes progressively shorten, causing the cell to lose its capacity to divide due to the loss of essential genes, or the ends of chromosomes being recognised as DNA damage \{Wright, 1992201 /id\}. Telomeres of different chromosomes shorten at different rates, although a DNA damage signal which induces replicative senescence can be evoked when only a few or even a single telomere is too short. Thus telomere shortening and induction of a senescence-associated cell cycle arrest has a tumour suppressor function which cancer cells must overcome prior to immortalisation \{Campisi, 2013190 /id\}.

The importance of telomeres can be observed during acute telomere uncapping, leading to induction of cellular senescence. A complex of six telomere-specific proteins, 
called shelterin, bind to and protect chromosome ends \{de Lange, 2010221 /id\}. Mammalian shelterin comprises: TRF1 and TRF2, two related homodimeric proteins that bind to double stranded TTAGGG repeats \{Broccoli, 1997223 /id\}; POT1 that binds to single-strand TTAGGG repeats, crucial for telomere protection \{Loayza, 2003226 /id\}; TPP1 that is required for recruiting POT1 to telomeres \{Ye, 2004229 /id\}; TIN2 which binds to TPP1 and interacts with both TRF1 and TRF2. The binding of POT1 to telomeres is critically dependent upon the TPP1/TIN2 interaction while the TRF1 and TRF2 proteins anchor the shelterin complex on double strand TTAGGG repeats. The other component is Rap1, an ortholog of yeast Rap1. The shelterin complex is highly specific for telomeric TTAGGG repeats and proposed to affect the structure of telomeric DNA, leading to the formation of a lariat-like structure called a t-loop such that the end of the telomere is tucked in \{Griffith, 1999224 /id\}. Loss of TTAGGG repeats, either progressively through continuous division, or acutely through telomere uncapping, removes the protective shelterin complex, resulting in the activation of a DNA damage response that leads to senescence \{de Lange, 2010221 /id\}. The DNA damage response pathway involves ataxia telangiectasia mutated (ATM), ataxia telangiectasia and Rad3 related (ATR), classical non-homologous end-joining as well as homologous recombination \{Sfeir, $2012220 / \mathrm{id}$ \}. The rapid evolution of telomere proteins initially hindered the identification of orthologues from other species, but recent studies have shown that the shelterin complex is present in multiple phyla \{Linger, 2009370 /id\}. 


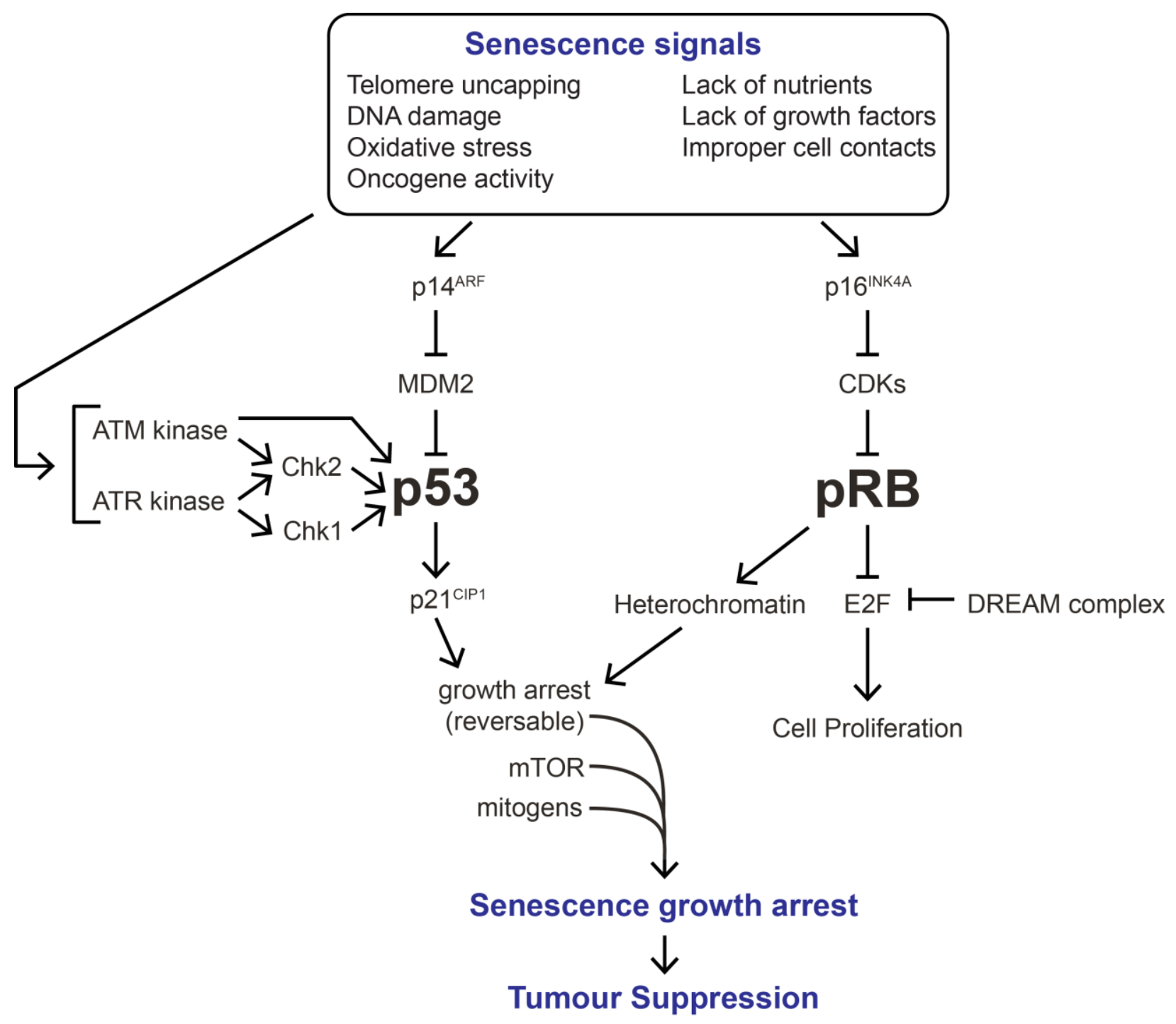

Figure 1. Senescence Triggers and Cellular Pathways to Senescence.

The two major pathways which lead to senescent growth arrest, p53/p21 and p16/pRB, are indicated. The p53/p21 pathway can be independently activated by either p14 ${ }^{\mathrm{ARF}}$ or the ATM/ATR kinases.

Other cell intrinsic and extrinsic stimuli can also induce senescence independently of telomere length by acting alone or in combination. Senescence-activating signals other than telomere uncapping include DNA damage, oxidative stress, oncogene activity, lack of nutrients, lack of growth factors and improper cell contacts (Figure 1). The finding that many diverse stimuli induce cellular senescence initially led to a distinction between "replicative senescence", which occurs following extended proliferation upon reaching 
the "Hayflick limit" (associated with telomere shortening) and "premature senescence", which occurs before the "Hayflick limit" and is triggered by intrinsic and extrinsic stressors \{Ben Porath, 2005184 /id\}. Senescence can also be induced in cancer cells, by some anti-cancer agents and radiation; this is described as therapy induced senescence \{Chang, 1999374 /id;Schwarze, 2005375 /id;Ewald, 2010372 /id\}.

Replicative senescence induced by extended proliferation is not entirely distinct from senescence driven by other inducers of senescence, such as telomere uncapping which also elicit a DNA damage response similar to other extrinsic stressors \{d'Adda, 2003 196 /id\}. Senescent human fibroblasts display molecular markers characteristic of cells carrying DNA double strand breaks, such as nuclear foci of phosphorylated histone $\mathrm{H} 2 \mathrm{AX}$ as well as activation of DNA repair and DNA damage checkpoint factors. The recruitment of DNA damage repair factors by the ends of chromosome ends, suggests that replicative senescence stems from the activation a DNA damage response by dysfunctional telomeres \{d'Adda, 2003196 /id\}. While recent studies have shown that "replicative" and "oncogene induced senescence" have many common changes in phenotype and gene expression compared to proliferating cells, they also exhibit substantial differences; this is also likely to be the case for other types of senescence \{Nelson, 2014204 /id\}.

The senescent phenotype is dominant over proliferation, as demonstrated by experiments involving fusion of normal human fibroblasts with different human cell lines, followed by determination of the proliferative capacity of the hybrids. The hybrids have limited proliferative potential, suggesting that the mortal state as indicated by entry into senescence is dominant and immortal cells arise as a result of recessive changes to overcome senescence \{Pereira-Smith, $1988213 / \mathrm{id}\}$. The fact that immortality is recessive was further exploited in experiments fusing immortal human cell lines with each other, to show that there are at least 4 complementation groups for indefinite division. These were not dependent upon cell type, embryonal layer of origin or the type of tumour from which they were derived \{Pereira-Smith, 1988213 /id\}. This further alludes to the senescence phenotype arising as an anti-tumour mechanism. 
Senescence has recently been shown to be a normal programmed event that occurs in mouse development. Storer et al \{Storer, 2013239 /id\} studied the apical ectodermal ridge and the neural roof plate, two signalling centres involved in embryonic patterning. They found senescence associated $\beta$-galactosidase (SA- $\beta$ gal) positive cells within the apical ectodermal ridge and showed that they exhibited features in common with oncogene-induced senescence. SA- $\beta$ gal activity is the most commonly used marker for senescent cells in vivo and in vitro \{Dimri, 1995368 /id\} due to them expressing acidic lysosomal $\beta$ - galactosidase \{Campisi, $2013190 /$ id\}. Munoz-Espin et al found that senescence occurs during mammalian development at multiple sites, including the mesonephros and the endolymphatic sac of the inner ear \{Munoz-Espin, 2013241 /id\}. They further found it was strictly dependent upon p21 ${ }^{\mathrm{CIP} 1}$, but independent of DNA damage and other cell cycle inhibitors. Together these studies proposed that senescence is a programmed mechanism that contributes to embryonic development and may be the evolutionary origin of the senescence program \{Munoz-Espin, 2013241 /id;Storer, 2013239 /id\}.

\section{Tumour Suppression}

The involvement of DNA damage response proteins in inducing senescence suggests a link between cellular senescence and cancer. Cancer cells accumulate genetic alterations which enable them to acquire a limitless proliferative potential, unlike normal cells, which lose the potential to divide at the "Hayflick limit" or upon exposure to stress. Early studies of oncogene action suggested that hyperactivity through dysregulation or overexpression results in increased cancer cell proliferation to fuel tumour growth \{Hanahan, $2011216 /$ id $\}.$ 
Although recent studies suggest that excessive oncoprotein expression promotes entry into senescence, or cell death by apoptosis, many cancers exhibit overactive oncogenes. For example, AKT hyperactivation, caused by excessive upstream signalling, or mutations in the AKT gene leading to its constitutive activation, have been implicated in the formation and progression of several human malignancies including breast, gastric and ovarian cancers \{Shukla, 2007232 /id\}. Another example is somatic gain-of-function mutations in the Ras family of genes, the first genetic alteration to be identified in human cancer. Since their identification, Ras proteins have been found to be essential components of various signalling pathways controlling cell proliferation, differentiation and survival, which are disrupted upon oncogenic mutation leading to tumour development \{Fernandez-Medarde, 2011233 /id\}. The finding that cancers can still develop despite complex multicellular organisms having senescence and apoptosis as key tumour suppressor mechanisms, indicates that a single mutation leading to oncogene activation is unlikely to be sufficient for oncogenesis, further strengthening the multi-step hypothesis of cancer progression \{Hanahan, 2011216 /id\}.

Unlike cellular senescence, apoptosis kills and completely eliminates potentially oncogenic cells when extrinsic or intrinsic stress signals activate pathways that lead to the release of cytochrome $\mathrm{c}$ from the mitochondria; thereby initiating a cascade of events leading to the activation of executioner caspases (cysteine-dependent aspartatespecific proteases). By cleaving their target proteins at key aspartate residues, activated executioner caspases cleave numerous different substrates resulting in the phenotypic changes seen during apoptosis $\{$ Tait, 2010234 /id\}.

Stressors, such as oncogene activation, can evoke either cell death or cellular senescence as a tumour suppressive response in multicellular organisms, since both lead to removal of damaged cells from the pool of proliferating cells. Cell death can be by apoptosis (programmed cell death) or by autophagy, a lysosomal degradation pathway \{Chen, 2011373 /id\}. Proliferating cells usually undergo growth arrest upon 
detection of DNA damage, thereby halting the cell cycle to allow DNA repair mechanisms to repair the damage before progressing through the cell cycle. When damage is too great or cannot be adequately repaired, mitotic cells rely on cell senescence or cell death to remove the cell from the proliferative pool to prevent the expansion of cells harbouring potentially oncogenic mutations \{Vicencio, 2008236 /id\}. However, the cell context is important, as cells may already be post-mitotic, or may have already acquired mutations that confer immortality or prevent cell death. It has been suggested that the choice between senescence and cell death depends upon the nature of inducing stimuli. For example, exposure of primary human diploid fibroblasts to 50 and $100 \mu \mathrm{M} \mathrm{H}_{2} \mathrm{O}_{2}$ predominantly induced senescence, whereas 300 and $400 \mu \mathrm{M}$ $\mathrm{H}_{2} \mathrm{O}_{2}$ mainly triggered apoptosis \{Bladier, $\left.1997237 / \mathrm{id}\right\}$. Concentration dependent induction of apoptosis and senescence are also observed when HT1080 fibrosarcoma cells and MCF-7 breast cancer cells are treated with DNA damaging agents, such as doxorubicin and epirubicin (14, \{Khongkow, 2014378 /id\}.

The senescence /cell death pathways may overlap to ensure that they are able to compensate for one another in the event that mutations remove the ability of cells to, either induce apoptosis, or enter into senescence. For instance, the secreted protein IGFBP7 has been identified as being necessary for an activated BRAFV600E oncogene to block proliferation in human primary fibroblasts and melanocytes. IGFBP7 is central to both senescence and apoptosis by BRAF; a negative feedback mechanism between IGFBP7 and ERK signalling leads to senescence-associated cell cycle arrest, whilst the activation of the pro-apoptotic BNIP3L protein downstream of IGFBP7 leads to induction of apoptosis \{Wajapeyee, 2008245 /id\}. This suggests that the nature of activated downstream genes is vital in determining cell fate between senescence and apoptosis, while further understanding of the way in which this regulation occurs is central to the development of senescence as an anti-tumour therapy. 


\section{Cellular Senescence as a Tumour Suppressor Mechanism}

There is extensive evidence linking cellular senescence with tumour suppression. The persistence of cellular senescence throughout evolution, despite the deleterious traits associated with reduced tissue renewal and repair, suggests that it must have a strong evolutionary benefit \{Campisi, $2013190 /$ id $\}$. Cell division is essential for the survival of complex multicellular organisms that contain renewable tissues, but cell division puts these organisms at risk of developing cancer - a hyperproliferative disorder. This has led to the hypothesis that cellular senescence evolved as a mechanism to halt proliferation of cells at risk of oncogenic transformation \{Campisi, 2013190 /id\}.

Many of the deleterious traits associated with a reduced potential for renewal and repair manifest in later life when the pool of actively dividing cells declines, suggesting that cellular senescence could have evolved if it provides an early-life benefit- a concept known as antagonistic pleiotropy \{Kirkwood, 2000247 /id;Campisi, 2013190 /id\}. Traits that manifest early in life are acted upon by a stronger force of natural selection than those which emerge at a later age, especially beyond the reproductive age. The major early-life evolutionary benefit of cellular senescence is the prevention of tumour formation in cells which acquire an expanded growth potential \{Kirkwood, 2000247 /id\}. In line with its evolutionary role, cellular senescence thus acts as a key tumour suppressor mechanism in adult multicellular organisms, but may have actually evolved from a primordial tissue - remodelling program active in embryonic development \{Munoz-Espin, 2013241 /id;Storer, 2013239 /id\}.

Most human and mouse cancers harbour mutations in key pathways implicated in senescence. A compromised ability to undergo senescence when induced is generally correlated with increased cancer susceptibility as deactivation of the senescence response has been observed to accelerate tumour formation in pre-malignant cells \{Collado, 2010194 /id\}. Studies in mouse models of oncogenic Ras expression have 
also shown that pre-malignant lesions contain a relative abundance of senescent cells, suggesting that entry into senescence is one of the mechanisms utilised by cells to prevent full malignant transformation \{Collado, 2010194 /id\}. Additionally, genetic manipulations, which induce premature senescence of mouse mammary epithelial cells, have been shown to suppress breast cancer formation in young mice exposed to a mammary tumour virus, further supporting the role of senescence in suppressing tumour formation in vivo \{Boulanger, 2001185 /id\}.

Consistent with its role in suppressing tumour formation in vivo, entry into cellular senescence is regulated by a plethora of stimuli that result in the activation of two central signalling pathways, the p53 and pRB tumour suppressor pathways (Figure1) \{Sedivy, 2013206 /id\}. Sometimes referred to as the p53/p21 and p16/pRB pathways, they are essential for establishing and maintaining senescence growth arrest in response to diverse intracellular and extracellular stimuli \{Adams, 2009248 /id\}. These two pathways interact, but can also initiate growth arrest independently by acting on different cell cycle regulatory proteins such as cyclin-dependent kinases (CDKs) \{Adams, 2009248 /id\}.

\section{The Senescence-Associated Secretory Phenotype}

In principle, entry into senescence is a tumour suppressor mechanism because protumorigenic cells are removed from the proliferating pool and thus cannot develop into a tumour. However, the situation is far more complex due to the senescence-associated secretory phenotype (SASP), the altered secretory profile of senescent cells which can affect the behaviour of non-senescent cells in the microenvironment \{Campisi, 2013190 /id\}. Senescent cells secrete a plethora of soluble signalling factors, such as inflammatory cytokines, chemokines and growth factors, matrix metalloproteinasetumour and extracellular matrix components known as the SASP 
proteins \{Kuilman, 2009251 /id;Coppe, 2010195 /id\}. The presence of these factors leads to shedding of membrane-associated proteins, degradation of signalling molecules and/ or degradation of the extracellular matrix comprising the basement membrane \{Kuilman, 2009251 /id;Coppe, 2010195 /id\}.

Whilst suppressing tumour formation in the senescent cell, the SASP proteins may actually promote tumour progression in neighbouring cells. The inflammatory cytokines IL-6 and IL-8 stimulate invasion of neighbouring tissues through the basement membrane by pre-malignant and malignant epithelial cells, thus promoting the formation of more aggressive metastatic tumours \{Karin, 2009258 /id\}. Matrix metalloproteinases (MMPs) are another component of the SASP, which degrade the basement membranes as well as various matrix proteins to allow escape of tumour cells from their site of initiation into the circulation thereby promoting metastasis \{Coppe, 2010195 /id\}. Apart from the mechanical alteration of the basement membrane, SASP components, such as the vascular-endothelial growth factor (VEGF), promote angiogenesis to help cancer cells overcome another key barrier to tumour

progression, the lack of nutrients and oxygen \{Coppe, 2010195 /id\}.

One of the key activators of the SASP is the nuclear factor kappa-light-chain-enhancer of activated B-cells (NF-kB) family of ubiquitously expressed transcription factors, important for the cellular response to stress $\{$ Hayden, 2008255 /id\}. This family of proteins regulate innate and adaptive immunity, which has a pro-tumorigenic side-effect \{Pikarsky, 2004257 /id;Karin, 2009258 /id\}, but have recently been shown to promote entry into senescence \{Freund, 2011259 /id;Rovillain, 2011270 /id\}. These opposite effects of NF-kB may depend on a variety of factors, including: the subunit expressed, the particular biological context and the nature of the upstream signal, which leads to activation of this pathway \{Vaughan, 2011254 /id;Mowla, 2013253 /id\}. 
Although senescent cells cannot be readily induced to re-enter the cell cycle, they remain metabolically active and exhibit an altered secretory profile which forms the SASP. This could contribute to the increase of cancer incidence as we age due to a build-up of senescent cells, as components of the SASP affect the pathophysiology of age-related diseases. Senescent cells have been shown to accumulate in the mitotic tissue of ageing primates \{Herbig, 2006377 /id;Jeyapalan, 2007376 /id\}. As the range of SASP functions is diverse, they could antagonize one another due to having pro- and anti-tumorigenic effects. The temporal organization of the SASP could also lead to differential SASP profiles at different time-points, leading to different and sometimes opposing downstream effects. For example, the same pro-inflammatory cytokines, which promote tumour progression early in senescence, also contribute to increasing immune clearance at later stages, thus further reinforcing the tumour suppressive effect of cellular senescence \{Campisi, $2013190 /$ id .

\section{Tumour Suppressor Genes Implicated in Cellular Senescence}

\section{p53}

Senescent growth arrest is initiated and maintained primarily through activation of the p53/p21 and p16/pRB tumour suppressor pathways. The genes encoding components of these pathways are all important in regulating cellular senescence \{Adams, 2009248 /id\}. The involvement of these key tumour suppressor genes is consistent with the evolutionary role of senescence, which is to prevent malignant transformation of cells harbouring irreparable DNA damage \{Campisi, 2013190 /id\}.

Known as the "Guardian of the genome", p53 plays a central role in preventing tumorigenesis through its functions in cell cycle regulation and maintenance of DNA integrity \{Amundson, 1998357 /id;Albrechtsen, 1999356 /id;Liu, 2001358 /id\}. Various types of cellular damage invoke rapid induction of high p53 levels in the cell, which lead 
to growth arrest to enable repair. In situations where the damage is too severe to repair, p53 activates alternative pathways which can lead to cellular senescence or cell death \{Amundson, 1998357 /id;Albrechtsen, 1999356 /id;Liu, 2001358 /id\}. This ensures that cells harbouring DNA damage are not permitted to proceed through the cell cycle and accumulate mutations, which could result in the cells becoming tumorigenic. As such, elevated p53 levels are associated with cells subjected to a wide range of stressors, including DNA damage, hypoxia, telomere shortening and oncogene activation, all of which are known to trigger senescence \{Amundson, 1998357 /id;Albrechtsen, 1999356 /id;Liu, 2001358 /id;Rufini, 2013264 /id\}.

The primary mechanism through which p53 levels are regulated in cells is by continuous degradation as a result of its binding to its principal antagonist mouse double minute 2 homolog (MDM2). MDM2 is an E3 ubiquitin ligase, which ubiquitinates p53 to promote its degradation by the proteasome \{Hock, 2014267 /id\}. MDM2 also directly blocks the transcriptional activity of p53 by binding to its $\mathrm{N}$-terminal transactivation domain Hock, 2014267 /id\}. MDM2 is a transcriptional target of p53, forming a negative regulatory loop whereby p53 induces the expression of its own negative regulator \{Hock, 2014267 /id\}. When cells are exposed to stress, MDM2 inhibition of p53 is removed, thereby activating p53 and increasing its level and its activity through post-transcriptional modifications. The two key mechanisms, used to couple stress and removal of MDM2 inhibition, is through phosphorylation by a signalling cascade initiated by the ATM kinase $\{$ Banin, $1998183 / \mathrm{id}\}$ and direct interaction with the CDKN2A protein (p14 ${ }^{\mathrm{ARF}}$ ), which is also encoded by the INK4 locus \{Pomerantz, 1998289 /id;Zhang, 1998287 /id\}. The latter mechanism is particularly interesting in the context of senescence because the same INK4 locus also encodes the p16 ${ }^{\text {INK4A }}$ cyclin-dependent kinase inhibitor (CDKI), central to evoking senescence- associated cell cycle arrest via the p16/pRB pathway. 
Another antagonist of p53 function is the MDM4/MDMX protein which is structurally similar to MDM2 and sequesters p53 in a similar manner. Moreover, both can bind to the $\mathrm{N}$-terminal transactivation domain of $\mathrm{p} 53$, thereby directly inhibiting its transcriptional activity by blocking binding of co-activators and recruiting repressors \{Hock, 2014267 /id\}. However, despite their similarities in interaction with p53, MDM4 has no intrinsic E3 ubiquitin ligase activity but MDM2 and MDM4 heterodimerize through their RING finger domains \{Hock, 2014267 /id\}. Although MDM2 and MDM4 can regulate p53 independently, there is growing evidence that the MDM2/MDM4 complex constitutes the principal E3 ubiquitin ligase for p53 \{Wang, 2011290 /id\}.

ATM kinase is a member of the phosphatidylinositol-3-kinase (PI-3K) family of kinases, which activates p53 through direct phosphorylation of Ser-15 and indirect phosphorylation of Ser-20 via Chk2 \{Banin, 1998183 /id\}. Chk2 may also activate p53 independently of ATM kinase possibly via the ATR kinase \{Hirao, 2002293 /id\}. Phosphorylation of p53 at key serine residues especially Ser-15, reduces the affinity of the p53-MDM2 interaction, leading to p53 stabilisation and elevated p53 levels. Serine phosphorylation is also required for $\mathrm{C}$-terminal acetylation of lysine residues required to stabilise p53 and enhance sequence-specific DNA binding.

The second mechanism of releasing p53 inhibition is through p $14^{\mathrm{ARF}}$ which increases in level following aberrant stimulation by upstream hypoproliferative signalling pathways, including activated Ras and c-Myc \{Sherr, $2001300 /$ id $\}$. P14 ${ }^{\text {ARF }}$ activates p53 by directly interacting with MDM2 and preventing it from targeting p53 for degradation \{Pomerantz, 1998289 /id;Zhang, 1998287 /id\}. Even though p14 ${ }^{\text {ARF }}$ and MDM4 are thought to not interact directly $\{$ Wang, $2011290 / \mathrm{id}\}$, it potentiates the interaction between MDM2/MDM4 leading to degradation of MDM4 and p53 activation \{Pan, 2003 $299 /$ id\}. 


\section{Key Cellular Functions of p53}

The best-characterised p53 activity is as a master transcriptional regulator, which induces or represses a plethora of genes involved in cell cycle arrest and DNA repair, effectively pausing the cell cycle to permit repair of DNA lesions \{Beckerman, 2010302 /id\}. In the context of senescence, an important p53 target is the cyclin-dependent kinase inhibitor $\mathrm{p} 21^{\mathrm{ClP} 1}$, which is required for senescence growth arrest in response to DNA damage at either the $G_{1} / S$ or the $G_{2} / M$ cell cycle checkpoints \{Brugarolas, 1995 304 /id;Waldman, 1995303 /id;Bunz, 1998188 /id\}. Mouse embryo fibroblasts null for p2 ${ }^{\mathrm{ClP} 1}$ are unable to undergo a radiation induced growth arrest \{Brugarolas, 1995304 /id\}. The role of $\mathrm{p}_{21}{ }^{\mathrm{CIP} 1}$ in $\mathrm{p} 53$-dependent $\mathrm{G} 1$ arrest was also demonstrated by comparing isogenic human colon adenocarcinoma cell lines differing only in their p2 ${ }^{\mathrm{ClP} 1}$ status. It was observed that the parental cell line underwent the expected cell cycle changes upon induction of p53 by DNA damage, but the $\mathrm{G}_{1}$ arrest was abrogated in the p21 ${ }^{\text {ClP1 }}$-deficient cells $\{$ Waldman, 1995303 /id\}. They further showed that cells harbouring DNA damage can undergo a $\mathrm{G}_{2}$ arrest independently of $\mathrm{p} 53$ and $\mathrm{p} 21^{\mathrm{CIP} 1}$; these cells however remain capable of undergoing mitosis, demonstrating that both p53 and $\mathrm{p} 21^{\mathrm{CIP} 1}$ are essential for maintaining a stable $\mathrm{G}_{2}$ arrest \{Bunz, $\left.1998188 / \mathrm{id}\right\}$ that may also be associated with senescence \{Gonos, 1996305 /id\}.

In addition to oncogenic signals and DNA damage, another regulator of senescence is the level of oxygen. Although atmospheric oxygen levels are around $20 \%$, the levels of oxygen in the body are lower and vary widely within organs. Most studies on senescence have been performed on cells cultured under standard tissue culture conditions utilizing $20 \%$ oxygen. Mouse embryo fibroblasts cultured in $20 \%$ oxygen undergo senescence whereas they proliferate indefinitely when cultured in $3 \%$ oxygen \{Parrinello, $2003306 /$ id\}. However there may be differences in the sensitivity of human and mouse cells to oxygen levels; there may even be further differences depending on the strain and the cell type eg. fibroblasts versus epithelial cells. Supraphysiological levels of oxygen give rise to oxidative stress through production of mitochondrial 
reactive oxygen species (ROS); other inducers of senescence, such as activated oncogenes and ionizing radiation, also increase intracellular ROS. ROS-mediated cell damage has been proposed to have a causative role in senescence and in ageing, in accordance with the free radical theory of ageing \{Barja, 2013366 /id\}.

Other studies however suggest that the link between hyperoxia, ROS and senescence may be more complex \{Welford, $2011308 /$ id $\}$. P53 can potentially inhibit or promote senescence by regulating ROS levels. It can repress senescence by lowering ROS levels by upregulating mitochondrial superoxide dismutase 2 , glutathione peroxidase and sestrins 1 and 2 \{Polyak, 1997312 /id;Hussain, 2004313 /id\}. In contrast, in cells that are highly sensitive to p53-mediated apoptosis, activated p53 can lead to a spike in ROS, resulting in cell death or senescence \{Lu, 2008315 /id;Macip, 2003316 /id\}.

Based on the function of p53 as a transcription factor, early research into the role of p53-dependent cellular senescence in tumour suppression has focussed on the mechanisms that trigger cell cycle arrest in response to acute DNA damage and identified key transcriptional targets including p2 ${ }^{\mathrm{CIP} 1}$. However, more recent studies have suggested that these targets are dispensable for tumour suppression. Knock-in mouse strains, expressing p53 mutants severely compromised for transcriptional activation of known target genes cannot induce $\mathrm{G}_{1}$ arrest or apoptosis in response to acute DNA damage. However they retain tumour suppressive properties, although p53 mutants which were completely defective for transactivation failed to suppress tumour formation \{Brady, 2011187 /id\}. This suggests that, in addition to mediating cell cycle checkpoints governing cell division, p53 induces cellular senescence by upregulating other novel target genes \{Brady, 2011187 /id\}. 


\section{Metabolism, p53 and Tumour Suppression}

Altered metabolism is a key change during cancer, allowing pre-cancerous cells to switch from a metabolic reliance on the tricarboxylic acid cycle in the mitochondria to the much more rapid glycolysis followed by lactic acid fermentation in the cytosol, known as "The Warburg Effect". The importance of this metabolic programming has recently been acknowledged as a "hallmark of cancer" \{Hanahan, 2011216 /id\}, although metabolism-related pathways such as insulin signalling through the mechanistic target of rapamycin (mTOR) pathway have previously been associated with a range of age-related diseases \{Johnson, 2013369 /id\}. The link between metabolism and age-related dysfunction is in accordance with Blagosklonny's theory of "hyperfunction", which suggests that ageing is a quasi-programme that is the consequence of a continuation of the processes involved in growth and development in early life \{Blagosklonny, 2013320 /id\}. When growth ceases, related pathways such as the nutrient-sensing mTOR continue to operate but now drive cellular and organismal aging \{Blagosklonny, 2013320 /id\}.

P53 interacts with mTOR and AMP-activated protein kinase (AMPK), two key regulators of cellular metabolism. MTOR forms two multiprotein complexes with distinct functions; mTORC1 controls cell growth and energy metabolism whereas mTORC2 regulates cell survival and the cytoskeleton. MTORC1 is active when conditions are favourable for cell growth, but is inhibited by cellular stress and inadequate growth conditions. It is regulated by a number of important signalling pathways including the IGF/PI3K/AKT growth factor signalling and p53-stress signalling. It can also be negatively regulated by energetic stress upon activation of AMPK, the cellular fuel sensor, which responds to increases in the AMP to ATP ratio. Blagosklonny and colleagues have shown that mTOR activity in the context of activated p53 is important in determining the outcome of the p53 response (Figure 2) \{Blagosklonny, 2012318 /id\}. Cells that sustain mTOR activity undergo geroconversion, a process that converts a reversible cell cycle arrest to a senescence arrest due to inappropriately high level of growth promotion (75-77). 
However cells in which mTOR is inhibited undergo reversible quiescence arrest \{Korotchkina, 2010323 /id;Xu, 2014367 /id\}. They further demonstrated that geroconversion can be suppressed by rapamycin \{Demidenko, $2009321 / \mathrm{id}$ \} and hypoxia \{Leontieva, 2012322 /id\}.

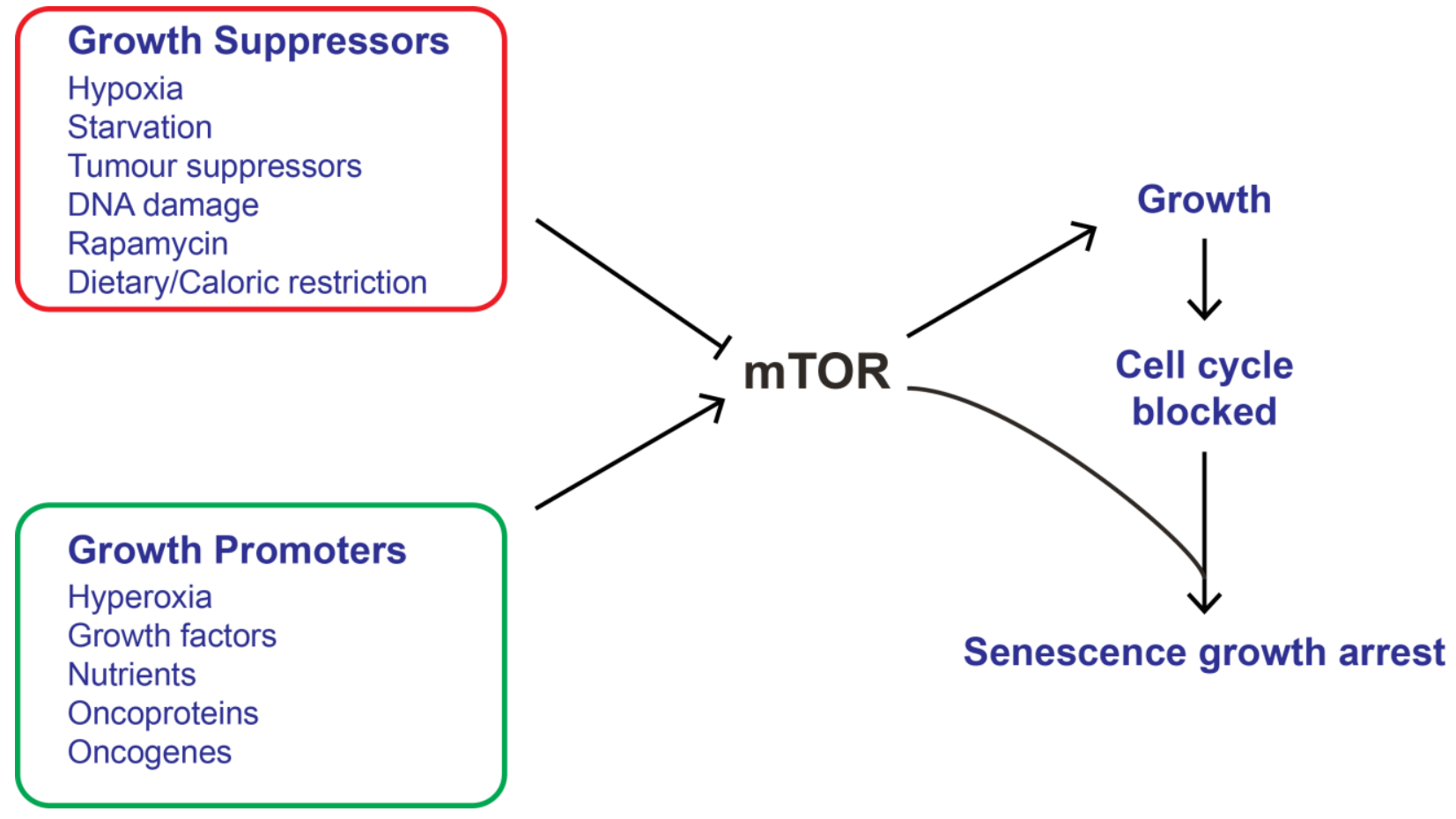

Figure 2. Mechanistic target of rapamycin (mTOR).

MTOR is a key protein which governs a cell's choice between division and arrest (72). Its activity is governed by growth suppressors and growth promoters which can either be intracellular or extracellular. Its activity is important in the context of activated p53 in determining the outcome of the p53 response (74). Cells that sustain mTOR activity undergo a stable senescence growth arrest, whereas cells in which mTOR is inhibited undergo a reversible quiescence arrest $(75,76)$.

Over the last decade, many studies have demonstrated that p53 counteracts cancerassociated metabolic transformation at various points in the metabolic network, such as in the surveillance of NADPH, which is produced by a range of metabolic enzymes. $\mathrm{NADPH}$ is critical for metabolism, as it is necessary for lipogenesis and facilitates production of reduced glutathione, for protecting tumour cells from ROS-induced damage. Previously Jiang et al \{Jiang, 2011327 /id\} implicated p53 in restricting 
NADPH production by inhibiting glucose flux through the pentose phosphate pathway. More recently, they have shown that p53 represses the expression of tricarboxylic acid cycle- associated malic enzymes ME1 and ME2 which are important for NADPH production, lipogenesis and glutamine metabolism \{Jiang, 2013317 /id\}.

Downregulation of ME1 and ME2 reciprocally activates p53 through MDM2 and AMPactivated protein kinase in a feed-forward regulatory loop \{Jiang, 2013317 /id\}. More interestingly, activation of p53 through down regulation of these malic enzymes modulates the outcome of p53 activation, strongly leading to induction of senescence, but not apoptosis; whereas enforced expression of either malic enzyme suppresses senescence \{Jiang, 2013317 /id\}.

\section{pRB}

The retinoblastoma protein (pRB), also known as the "Guardian of the Restriction Point", is a key tumour suppressor that exerts its effects on the cell cycle through an inhibitory action on E2F transcription factors. It is a member of the family of pocket proteins that share a pocket domain originally identified as being required for binding viral oncoproteins, such as adenovirus E1A, SV40 large T antigen and human pappiloma virus E7 \{Dick, 2013339 /id\}. Members of the retinoblastoma family of proteins include pRB, p107 and p130; they are homologous within the pocket domain and have a similar domain structure which is consistent with their analogous cellular functions \{Dick, $2013339 /$ id\}. However there are key functional differences between them: for example, the tumour suppressor functions of $\mathrm{pRB}$ are stronger than those of p107 and p130, since only pRB is commonly found to be mutated in human cancer \{Helmbold, 2012332 /id\}.

pRB has long been recognized as a crucial mediator of the $G_{1} / S$ cell cycle checkpoint. In $G_{0}$ and $G_{1}$ phases of the cell cycle, pRB is present in its active dephosphorylated form and binds to the heterodimeric E2F transcription factors, to inhibit E2F-dependent 
gene expression required for cell cycle progression and DNA replication \{Lowe, 2003 346 /id\}. pRB binds preferentially to activating E2Fs (E2F1, E2F2 and E2F3), in association with their dimerization partners (DPs), to repress their activity by blocking the transactivation domain and recruiting other proteins, such as histone deacetylases and chromatin remodellers, to promote heterochromatin formation to repress transcription \{Weintraub, 1995343 /id\}. PRB binds to the E2F transactivation domain via a highly conserved region of the pocket domain. The pocket domain also contains the LXCXE binding cleft which is bound by the LXCXE motif present within pRBinteracting proteins such as the viral oncoproteins. These viral proteins functionally inactivate $\mathrm{pRB} / \mathrm{p} 107 / \mathrm{p} 130$ by sequestering them.

Most of the effects of pRB on the cell cycle are exerted during the $G_{1}$ phase, where cells entering this phase from quiescence require stimulation by mitogens until just before the onset of the S phase after which the cell cycle becomes mitogen-independent. This decision point is known as the Restriction $(R)$ point \{Pardee, 1974342 /id\}, and cells which pass the $\mathrm{R}$ point commit to the remainder of the cell cycle barring major DNA damage or metabolic disturbance. Progression of the cell through early $\mathrm{G}_{1}$ to late $\mathrm{G}_{1}$ and past the $R$ point is dependent on the phosphorylation state of pRB \{Weinberg, 1995 344 /id\}.

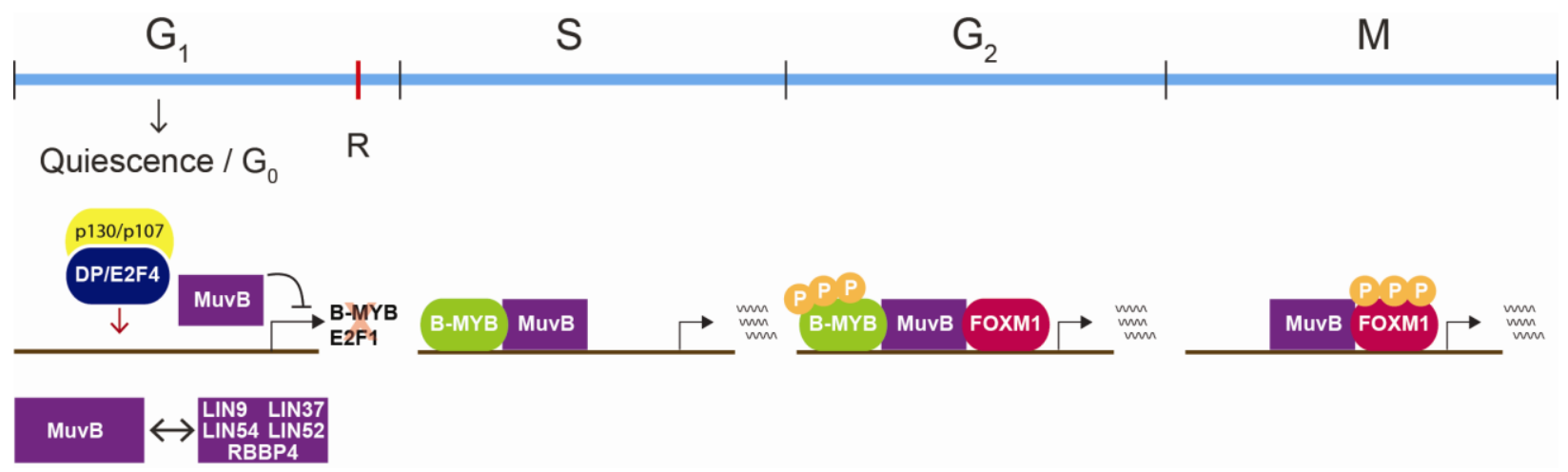

Figure 3. Regulation of cell cycle gene expression by MuvB complexes.

The MuvB complex comprises five MuvB-like proteins: LIN9, LIN37, LIN52, LIN54 and RBBP4. It regulates gene expression at different stages of the cell cycle by associating 
with different factors. In quiescence, MuvB associates with p130/p107, E2F4 and DP to from the DREAM complex which represses cell cycle dependent gene expression (92, 93). When cells exit quiescence, p130 dissociates from MuvB and from E2F-dependent promoters, enabling activator E2Fs to transactivate genes required for $S$ phase (93). MuvB then associates with B-MYB to regulate late $S$ phase genes. During G2, the MuvB-B-MYB complex recruits FOXM1, to these promoters $(92,93)$. This is followed by phosphorylation-dependent proteasome-mediated degradation of B-MYB and activation of FOXM1 by phosphorylation. Hyperphosphorylated FOXM1 remains bound to the MuvB complex and modulates expression of genes required for the G2-M transition (93). The role of the DREAM complex in senescence remains to be fully characterized but both B-MYB and FOXM1 are lost from the MuvB complex which then recruits the p130/p107-E2F4-DP complex, to form the transcriptionally repressive DREAM complex.

Different members of the cyclin dependent kinase family govern different stages of the cell cycle. PRB contains 16 putative CDK-phosphorylation sites which allow phosphorylation in early $\mathrm{G}_{1}$ by $\mathrm{CDK} 4 / 6$. However before $\mathrm{CDK}_{4} / 6$ is able to phosphorylate $\mathrm{pRB}$, it needs to associate with cyclin $\mathrm{D}$ which is transcriptionally activated by mitogens to produce an active kinase complex. This level of phosphorylation is however insufficient to disassemble the repressive complexes formed by the $p R B$ family of proteins. Late in $G_{1}$, further phosphorylation of $p R B$ by CDK2 in complex with its binding partner cyclin E, leads to disruption of HDAC-pRBE2F complexes, allowing activation of E2F-responsive genes for progression of cells through $G_{1}$ and entry into $S$ phase $\{$ Weinberg, $1995344 / i d\}$. This canonical model of tumour suppression by $\mathrm{pRB}$, based on inhibition of E2F dependent transcription, does not explain how p107 and p130 inhibit the cell cycle by binding E2Fs that repress transcription. 


\section{Interaction with p53 in Cellular Senescence}

The physiological role of pRB in the control of the cell cycle allows this tumor suppressor protein to play an important role in establishing the cell cycle arrest associated with cellular senescence. DNA damage response-related stimuli primarily induce senescence via activation of p53/p21, whilst non-genotoxic stress such as oncogenic Ras signalling or tissue culture stress induce senescence through the p16/pRB pathway by inhibiting CDK4/6 activity through p16 ${ }^{\text {INK4A }}$ activation $\{$ Lin, 1998 360 /id;Lowe, 2003346 /id\}.

There is cross talk between the p53/p21 and p16/pRB pathways, since p53 activation increases levels of $\mathrm{p} 21^{\mathrm{ClP} 1}$, which can inhibit hyperphosphorylation of $\mathrm{pRB}$ proteins by cyclin E-CDK2, required to release inhibition of E2F transcription factors for traverse of $\mathrm{G}_{1}$; this further enforces senescence growth arrest. P21 ${ }^{\mathrm{CIP} 1}$ can also arrest cell cycle progression by inhibiting cyclin A- and cyclin D-dependent kinase complexes \{Vaughan, $2011254 / \mathrm{id}\}$.

The importance of pRB in maintaining a stable cell cycle arrest in senescence has been demonstrated by acute inactivation in senescent cells. Senescent cells upon pRB inactivation attempt to renter $\mathrm{S}$ phase and reinitiate DNA synthesis but undergo an E2Fdependent apoptosis due to activation of p73, a member of the p53 family of proteins \{Alexander, $2003178 / \mathrm{id}$ \}. This demonstrates that functional pRB is not only required for the establishment of senescence, but is required to maintain it \{Alexander, 2003178 /id\}. Furthermore Hara and colleagues have now shown that the switch from quiescence to senescence, upon activation of $\mathrm{pRB}$, requires the presence of mitogens to induce ROS \{Imai, $2014350 /$ id $\}$.

The exact role of the different members of the pRB family remain unclear, although some studies have suggested that there is a non-redundant role for each of these proteins and that $\mathrm{p} 130$ is the only one that is indispensable for the maintenance of senescence growth arrest. Chicas et al have shown that $\mathrm{pRB}$ represses a unique group of genes required for senescence but not any other growth states \{Chicas, 2010193 
/id\}. Whilst the shift in the phosphorylation state of $p R B$ is critical for the initial rapid response to DNA damage, leading to transient arrest, at this stage the cells do not show senescence markers and can escape the cell cycle arrest \{Kapic, 2006345 /id\}. Cells which remain arrested and progress into senescence undergo changes in pocket protein expression where they switch from pRB to p130 expression. These cells typically exhibit decreased levels of pRB and p107 upon senescence, whilst the levels of p130 increase around the time that phenotypic markers of senescence appear and the cell cycle arrest becomes irreversible \{Helmbold, 2012332 /id\}.

\section{The DREAM Complex}

Recent studies by DeCaprio and colleagues\{Sadasivam, 2013340 /id\} have found that p130 and p107 form a multi-subunit DREAM complex upon association with dimerization partner (DP), E2F and a multi-vulval class B (MuvB) core complex, made up of five MuvB-like proteins LIN9, LIN37, LIN52, LIN54 and RBBP4 (Figure 3). The DREAM complex represses cell cycle-dependent genes during quiescence, and for the cell cycle to proceed, the MuvB core must dissociate from p130 and bind to B-MYB to form the B-MYB-MuvB complex which activates genes expressed later in $S$ phase. This complex recruits FOXM1 during $\mathrm{G}_{2}$, enabling FOXM1 to activate expression of genes important for the $\mathrm{G}_{2} / \mathrm{M}$ transition \{Sadasivam, 2012348 /id;Sadasivam, 2013340 /id\}. The MuvB core is essential for targetting B-MYB and FOXM1 to the promoters of specific cell cycle dependent genes \{Sadasivam, 2013340 /id\}. Although the role of the DREAM complex in senescence remains to be fully characterised, mutations which disrupt DREAM assembly have been found to suppress Ras-induced senescence \{Litovchick, 2011341 /id\}.

\section{P16 ${ }^{\text {INK4A }}$}

P16 ${ }^{\text {INK4A }}$ is a member of the INK4 (inhibitors of CDK4) family of CDKIs that was originally isolated as a protein that specifically blocks the activity of cyclin D-CDK4 
kinase $\left\{\right.$ Serrano, 1993351 /id\}. Members of this family include $\mathrm{p} 16^{\mathrm{INK} 4 \mathrm{~A}}, \mathrm{p} 15^{\mathrm{INK} 4 \mathrm{~B}}$, $\mathrm{p} 18^{\mathrm{INK} 4 \mathrm{C}}$ and $\mathrm{p} 19^{\mathrm{INK} 4 \mathrm{D}}$. INK4 inhibitors block CDK4/6 activity by distorting either the cyclin-binding site to reduce the affinity for D-type cyclins, or by altering the structure of the ATP binding site to compromise catalytic activity \{McConnell, 1999361 /id\}. Inhibition of cycD-CDK4/6 complexes prevents phosphorylation of $\mathrm{pRB}$, thereby leading to cell cycle arrest in $\mathrm{G}_{1}$ \{Rayess, $\left.2012336 / \mathrm{id}\right\}$. P16 ${ }^{\text {INK4A }}$ maps to chromosome 9p21, a locus first discovered as being frequently mutated in familial melanomas (malignant melanomas) \{Cannon-Albright, 1992380 /id;Kamb, 1994379 /id;Kamb, 1994381 /id\}. P16 alterations have subsequently been observed in many other human cancers \{FitzGerald, 1996363 /id;Liggett, 1998364 /id\}. It is activated by non-genotoxic stress, such as inadequate culture conditions, activation of an oncogene or by oxidative stress \{Iwasa, 2003352 /id\} and particularly important for inducing senescence following p53 inactivation. It may act as a backup tumour suppressor gene for p53, as both of these proteins are able to induce senescence independently of one another \{Rayess, 2012 336 /id\}. The INK4A locus is very important in the regulation of cellular senescence because its two main gene products, $\mathrm{p} 16^{\mathrm{INK} 4 \mathrm{~A}}$ and $14^{\mathrm{ARF}}$ affect the two major pathways, p53/p21 and p16/pRB, for inducing senescence.

\section{Conclusion}

Cellular senescence provides one of the strongest anti-oncogenic mechanisms through key tumour suppressors, namely p53 and pRB that inhibit a variety of signalling pathways which act to induce growth, proliferation and tumorigenesis. We have described the role of these tumor suppressors in mediating several physiological processes that, when deregulated, lead to key hallmarks of cancer. Through these tumor suppressors, cellular senescence stringently inhibits these hallmarks such as replicative immortality, constitutive growth, metabolic shifts and inappropriate cell survival by deploying critical downstream targets such as CDKIs. Cellular senescence is therefore an evolutionary mechanism to suppress tumor formation and could thus potentially be utilised in the prevention and/or treatment of cancer, although it may 
actually have originated as a remodelling program active in embryonic development \{Longo, 2003354 /id;Munoz-Espin, 2013241 /id;Storer, 2013239 /id\}.

Apart from cell cycle arrest, a better understanding of SASP and how its effects can be modulated has recently been explored as another avenue for controlling cancer. For example, pharmacological targeting of NF- $\mathrm{kB}$ by metformin, a drug used to control diabetes mellitus, has been shown to inhibit the SASP \{Moiseeva, 2013355 /id\}. This has been demonstrated in human trials to be effective in the control of prostate cancer \{Azvolinsky, $2014179 / \mathrm{id}$ \}, suggesting that the modulation of senescence and its associated SASP can lead to novel ways of treating cancer. However, the complex nature of senescence and the diverse impact that the SASP has on the surrounding cells means that any treatment that makes use of this cellular mechanism needs to maintain the fine balance between anti- and pro-tumorigenic effects in order to yield the desired results. Further investigation of such conditions is vital to exploitation of cellular senescence in the treatment of cancer.

\section{Acknowledgements}

We apologize to authors whose work is not discussed / cited due to space limitations. We are indebted to Richard Newton for graphics. ASLC and SNM were "MSci students in Biological Sciences and Natural Sciences" at University College London. The authors report no conflict of interest in this work. 